\title{
Estrategias del plan nacional de control del cáncer y participación comunitaria
}

\author{
Herlinda Torres-Nieto*
}

\section{RESUMEN}

Introducción: el cáncer, enfermedad crónica de origen multifactorial no transmisible, se ha convertido en un problema de salud pública, lo que implica abordarlo desde los cambios sociodemográficos de la población y los determinantes sociales de la salud, para implementar estrategias de prevención primaria e intervenciones oportunas y coordinadas a nivel político, servicios de salud, con movilización social y participación comunitaria. Metodología: se realizó una revisión sistemática de 50 artículos científicos, documentos de normatividad y diferentes publicaciones acerca de los planes de control del cáncer a nivel mundial y regional, en el periodo del año 2010 al 2015. Las bases de datos utilizadas, Proquest, LILACS, los índices bibliográficos Scielo y la Biblioteca Virtual en Salud. Las palabras clave utilizadas fueron: cáncer, factores de riesgo y prevención primaria. Resultados: los planes de control de cáncer buscan integrar los sistemas de salud existentes para aplicar estrategias en forma transversal, tanto de prevención primaria como de tratamiento del cáncer. Conclusión: la promoción de la salud constituye una estrategia de difusión y de educación para la comunidad en general. Las organizaciones que apoyan a los pacientes con cáncer realizan una labor significativa, porque logran una movilización social importante frente a esta problemática de salud pública.

PALABRAS CLAVE: cáncer, enfermedad, promoción de la salud**.

\footnotetext{
* Enfermera.

Magister en

Salud Pública

y Desarrollo

Social. Docente.

Fundación

Universitaria del

Área Andina.

Bogotá D.C.

Colombia. Correo

electrónico:

hetorres3@

areandina.edu.co

**Descriptores en Ciencias de la Salud (DeCS), en la página http://

decs.bvs.br/E/

homepagee.htm

de la Biblioteca

virtual en salud del proyecto BIREME, de la Organización Mundial de la

Salud y de la

Organización

Panamericana de

Salud.
} 


\section{National plan strategies for cancer control plan and community participation}

\section{ABSTRACT}

Introduction: cancer, chronic noncommunicable multifactorial originating, has become a public health problem, which implies approaching it from the socio-demographic changes in population and social determinants of health, to implement primary prevention strategies and interventions timely and politically coordinated, health services with social mobilization and community participation. Methodology: a systematic review of 50 scientific articles, papers of regulations and different publications about cancer control plans at global and regional level in the period from 2010 to 2015 was conducted. Databases used, Proquest, LILACS, bibliographic indexes Scielo and Virtual Health Library. The keywords used were: cancer, risk factors and primary prevention. Results: cancer control plans, seek to integrate existing health systems, to implement strategies transversely both in primary prevention and treatment of cancer. Conclusion: health promotion is a strategy of outreach and education for the community in general. The organizations that support cancer patients, performs significant work, because they manage a major social mobilization against this public health problem.

KEY WORDS: cancer, disease, health promotion. 


\section{Estratégias plano nacional de controlo do cancro e de participação comunitária}

\section{RESUMO}

Introdução: câncer, origem multifatorial crônicas não transmissíveis, tornou-se um problema de saúde pública, o que implica abordá-lo a partir das mudanças sóciodemográficas da população e os determinantes sociais da saúde, a implementação de estratégias de prevenção primária e intervenções nível de serviços política, saúde atempada e coordenada, mobilização social e participação da comunidade. Metodologia: bancos de dados Uma revisão sistemática de 50 artigos científicos, artigos de regulamentos e diversas publicações sobre os planos de controlo do cancro a nível global e regional, no período de 2010 a 2015 foi realizado utilizados, a ProQuest, LILACS, índices bibliográficos Scielo e Biblioteca Virtual em Saúde. As palavras-chave utilizadas foram: câncer, fatores de risco e prevenção primária. Resultados: planos de controlo do cancro, procuram integrar sistemas de saúde existentes, a implementação de estratégias de forma transversal a prevenção e tratamento do câncer primário. Conclusão: a promoção da saúde é uma estratégia de sensibilização e educação para a comunidade em geral. As organizações que apóiam pacientes com câncer, realizar um trabalho significativo, porque eles conseguem uma grande mobilização social contra este problema de saúde pública.

PALAVRAS-CHAVE: cancro, doença, promoção da saúde. 


\section{INTRODUCCIÓN}

Ene - Jun 2016

Cúcuta, Colombia $\checkmark$ ste artículo describe las estrategias del Plan Nacional de Control del Cáncer en Colombia para el período 2012-2020 y se hace énfasis en la línea estratégica 1 , prevención primaria del cáncer, teniendo en cuenta que la Organización Mundial de la Salud (OMS) propone, en su plan de acción del control de las enfermedades no transmisibles, la reducción de los factores de riesgo modificables para crear entornos saludables y, al mismo tiempo, fortalecer la capacidad de las personas para adoptar comportamientos de vida saludables con el fin de fomentar la salud (1).

El cáncer forma parte de las enfermedades crónicas no transmisibles, según la Organización Panamericana de la Salud (OPS), junto con enfermedades cardiovasculares, diabetes y enfermedades respiratorias crónicas, que representan la mayor carga de enfermedad en la Región de las Américas (2).

La OPS plantea la estrategia para la prevención y el control de las enfermedades no transmisibles, en el período 2012-2025, y propone cuatro líneas de acción que incluyen políticas y alianzas a nivel multisectorial con análisis de factores de riesgo y factores protectores con vigilancia e investigación a nivel regional y nacional (2)

El cáncer es el resultado que presentan las células cancerosas en genes relacionados con el control del ciclo celular. El origen de estas mutaciones es, en la mayor parte de los casos, hasta el $84 \%$ el factor ambiental (3). El cáncer forma parte del grupo de enfermedades con grandes repercusiones sociales, económicas y emocionales; por tanto, incluye el ámbito comunitario, que fomenta una movilización social importante frente a una enfermedad de alto costo.

Además, el cáncer es una enfermedad crónica de origen multifactorial que se ha convertido en un problema de salud pública por la alta incidencia y la mortalidad que produce, así como el elevado costo social y económico que conlleva. La OPS define la salud pública "como la salud del público, de la población y abarca todas las dimensiones colectivas de la salud" (4).

El cáncer es una de las principales causas de morbilidad y mortalidad a nivel mundial. En el año
2012, se registraron 14 millones de nuevos casos y 8.2 millones de muertes por su causa. Según la OMS, se prevé que el número de casos nuevos llegue a un $70 \%$ en los próximos 20 años. E1 $70 \%$ de las muertes por cáncer que se registraron en el año 2012 ocurrieron en África, Asia, América Central y Sudamérica (5).

Los tipos de cáncer que más se diagnosticaron en los hombres, en el año 2012, fueron los de pulmón, próstata, colon, recto, estómago e hígado. En las mujeres fueron cáncer de mama, colon, recto, pulmón, cuello uterino y estómago. Los tipos de cáncer más frecuentes son diferentes en el hombre y en la mujer. Las tasas estandarizadas más elevadas de mortalidad en mujeres, por cáncer de mama, se registraron en los países del cono sur (Chile, Paraguay y Uruguay) y los países del Caribe inglés (Jamaica y Trinidad y Tobago) con más de 20 muertes por 100.000 mujeres; mientras que las tasas más bajas correspondieron a las observadas en Guatemala, El Salvador y Ecuador, con menos de 10 muertes por 100.000 mujeres (5).

Por su parte, las tasas estandarizadas de mortalidad por cáncer cérvico-uterino más altas se produjeron en Nicaragua, Paraguay y Perú, con valores medios de 16,2 muertes por 100.000 mujeres; y las tasas más bajas en Canadá, Estados Unidos y Puerto Rico (5).

En Colombia se registraron, en promedio, 16.081 muertes anuales en hombres y 16.572 en mujeres, en el periodo 2007- 2011. Para la población infantil, el tipo de cáncer con mayores tasas de mortalidad fue el de las leucemias, con 148 casos estimados en niños y 108 en niñas. Los departamentos con mayores tasas de mortalidad por cáncer infantil, en Colombia, fueron San Andrés, Atlántico, Norte de Santander y Risaralda (6).

En el país se presentan anualmente alrededor de 2.200 nuevos casos de cáncer infantil que, de ser diagnosticados de forma temprana, podrían ser curados. Según cifras del Instituto Nacional de Cancerología (INC), la tasa de incidencia de casos nuevos de cáncer infantil en el país es, en promedio, mayor a la Latinoamericana. En el caso colombiano, la primera causa de enfermedad y muerte por cáncer infantil está dada por las leucemias agudas, con 539 casos y 295 muertes cada año (6).

El Departamento Administrativo Nacional de Estadística (DANE), en el período de enero a agosto 
de 2015 , reportó que el $25,4 \%$ de los fallecimientos en mujeres fueron causados por algún tipo de cáncer. El Observatorio de Mujeres y Equidad de Género, en Bogotá, se unió a la celebración del día mundial de lucha contra el cáncer, el 4 de Febrero, para contribuir con la difusión del panorama real de morbi mortalidad a causa de esta enfermedad. (7)

Las infecciones que pueden provocar cáncer, como el virus de la hepatitis B y C y el Virus del Papiloma Humano (VPH), son responsables del $20 \%$ de las muertes por cáncer en los países de ingresos bajos y medios y del $7 \%$ de muertes en los países de ingresos altos (5).

Algunos tipos de cáncer podrían prevenirse evitando factores de riesgo como el tabaco, el consumo abusivo de alcohol, la dieta poco saludable y la inactividad física. El tabaquismo es el factor de riesgo que, por sí solo, provoca un mayor número de casos y a nivel mundial causa aproximadamente un $22 \%$ de las muertes por cáncer y un $71 \%$ de las muertes por cáncer de pulmón (5).

Los programas de tamizaje y vacunación representan intervenciones efectivas para reducir la carga de determinados tipos de cáncer. Muchos tipos de cáncer tienen una probabilidad de curación si se detectan a tiempo y se tratan adecuadamente (OMS, 2011).

\section{METODOLOGÍA}

Se realizó una búsqueda de artículos sobre planes de control de cáncer en el mundo, tema al cual no es fácil el acceso. Se efectuó una revisión sistemática de artículos científicos, documentos de normatividad y diferentes publicaciones acerca de los planes de control del cáncer, a nivel mundial y regional, en el periodo 2010 a 2015. Se revisaron 50 artículos; sin embargo, no todos estos artículos correspondieron al periodo señalado, pero se rechazaron los artículos anteriores al año 2002.

Las bases de datos utilizadas fueron Proquest, LILACS, los índices bibliográficos Scielo y la biblioteca virtual de salud. Para determinar la pertinencia, los artículos fueron revisados a partir del resumen y los libros se revisaron a partir de la tabla de contenido, en español e inglés, con las palabras clave: cáncer, factores de riesgo y detección temprana. Los artículos que se encontraron en mayor cantidad tenían que ver con tipos de cáncer en la mujer, cáncer de mama y cáncer cervico uterino.

\section{Factores de riesgo}

Un factor de riesgo es todo aquello que afecta la probabilidad de adquirir una enfermedad. Los diferentes tipos de cáncer tienen distintos factores de riesgo; sin embargo, los factores de riesgo no lo indican todo porque, si se tiene uno o varios factores, esto no significa que necesariamente se desarrollará la enfermedad. Existen diferentes factores de riesgo y algunos de ellos, como la edad, la raza y el envejecimiento no se pueden modificar. Otros están relacionados con factores cancerígenos ambientales (8).

Otros factores de riesgo tienen que ver con comportamientos personales, entre ellos el consumo de cigarrillo, el consumo de bebidas alcohólicas, la alimentación poco saludable, la falta de actividad física y conductas sexuales inseguras, los cuales son los factores de riesgo modificables y de mayor interés en el campo de la prevención primaria de las enfermedades crónicas no transmisibles, como el cáncer.

El cáncer comienza con la transformación de una sola célula, que puede tener su origen en agentes externos y en factores genéticos heredados. En esta enfermedad, las células se multiplican sin control con la probabilidad de invadir tejidos vecinos, viajar por el torrente sanguíneo y linfático y diseminarse por otras partes del organismo. En la aparición de la mayoría de los tumores malignos influyen factores ambientales, físicos, químicos, dieta con aditivos y conservantes, consumo de tabaco y alcohol, contaminantes ambientales y algunas infecciones. La identificación de estas causas ambientales genera la esperanza de la prevención del cáncer (3).

Dentro de los factores de riesgo para el cáncer se mencionan los antecedentes familiares, especialmente en el cáncer de seno, que se constituye en una preocupación a nivel mundial debido a la mortalidad que conlleva la detección avanzada de la enfermedad. En los Estados Unidos es la segunda causa principal de muerte de las mujeres; la primera causa la ocupa el cáncer de pulmón. Según las estadísticas y cálculos de la Sociedad Americana del Cáncer, una de cada 
ocho mujeres tiene riesgo de padecer cáncer de mama a lo largo de la vida y una de cada 28 mujeres tiene riesgo de morir por cáncer de mama (8).

En el cáncer de seno existen distintos tipos de riesgo. Muchas mujeres tienen uno o más factores de riesgo para cáncer de seno, pero nunca padecen la enfermedad, mientras que otras mujeres, sin aparentes factores de riesgo, desarrollan la enfermedad. En cuanto al factor hereditario, Romero et al. (9) presentan la revisión de 272 expedientes de pacientes con cáncer de mama, encontrando $6.6 \%$ de pacientes con antecedentes familiares en primer grado de consanguinidad de cáncer de seno y $9.2 \%$, que corresponde a 25 casos, presentó factores hereditarios de cáncer de mama en segundo grado de consanguinidad.

Respecto al factor de riesgo inactividad física para el cáncer de mama, Aguilar et al. (10) describen la asociación entre obesidad, sobrepeso e inactividad física con el cáncer de mama en una muestra de 115 mujeres, en un estado mexicano, en el año 2011.

En forma generalizada, un $30 \%$ de las muertes ocurridas por cáncer obedecen a factores de riesgo de conducta y dietéticos, entre ellos índice de masa corporal elevado, reducida ingesta de frutas y verduras, inactividad física, consumo de tabaco y consumo de alcohol (1).

El consumo simultáneo de alcohol y tabaco aumenta el riesgo de cáncer de las vías respiratorias y del tracto digestivo superior. Las personas que fuman y beben en forma simultánea aumentan considerablemente el riesgo de cáncer si se comparan con las que no lo hacen.

La política pública, desarrollada en la Ley 1355 del año 2009, define la obesidad como una enfermedad crónica de salud pública que causa enfermedades cardiacas, circulatorias, depresión, cáncer y diabetes, aumentando considerablemente la tasa de mortalidad de los colombianos. La promoción de entornos y hábitos saludables es fundamental, así como una alimentación saludable y balanceada hace parte de una política pública(11).

En los países de la Europa Occidental, el sobrepeso y la obesidad explican aproximadamente el $11 \%$ del total de casos de cáncer de colon, el $9 \%$ de los casos de cáncer de mama, el $39 \%$ de los de endometrio, el
$37 \%$ de los de esófago y el $25 \%$ de cáncer de riñón (12).

En los Estados Unidos, el $6 \%$ de todos los cánceres son orales y el $30 \%$ son en cabeza y cuello, muchos de los cuales provienen de cavidad oral. En Colombia, en el Instituto Nacional de Cancerología, se presentan anualmente entre 100 y 120 nuevos casos de cáncer oral, el $50 \%$ en lengua -3 veces más frecuente en hombres que en mujeres-; el $74 \%$ de los casos ocurre entre los 50 y 70 años. Se deduce, en este estudio, que la edad es el principal factor de riesgo en el desarrollo del cáncer oral(13).

Es importante resaltar que en las últimas décadas se ha venido produciendo un importante cambio en la estructura de las poblaciones, especialmente en países de Europa Occidental como España, lo cual coincide con el aumento de la población adulta mayor $\mathrm{y}$, adicionalmente, la exposición a factores de riesgo como el tabaquismo se presenta cada vez más en personas jóvenes. El cáncer oral puede dividirse en tres grupos: carcinomas propios de la cavidad oral, carcinomas de labio y carcinomas de la orofaringe (13).

Hoy día se encuentra una relación estrecha del virus del papiloma humano con la creciente exposición a carcinógenos como el tabaco y el alcohol. Respecto a la historia natural de la enfermedad se puede afirmar que las lesiones orales premalignas, como leucoplasia y eritroplasia, han sido consideradas las lesiones orales precancerosas por excelencia en todo el mundo(13).

La OPS describe la asociación entre algunos agentes virales y la producción de diferentes tipos de cáncer; es el caso de cáncer cervico uterino en asocio con virus del papiloma humano, carcinoma hepatocelular con el virus de la hepatitis $\mathrm{B}$ y $\mathrm{C}$, carcinoma gástrico con Helicobacter pylori (14).

En las ultimas 3 décadas se ha presentado de un 3 a $9 \%$ de prevalencia en cáncer de cabeza y cuello en México. En Colombia, este tipo de cáncer representa entre el 5 y el $10 \%$, según estudios realizados en los últimos años por el INC, 2010 (13).

Serena et al. (13) describen la asociación de infecciones virales con cáncer de cabeza y cuello; el cáncer bucal es el más frecuente con un 45,6\% 
(lengua $21 \%$, encía $5 \%$, piso de boca $2 \%$, labio $2,5 \%$, otras localizaciones de la mucosa oral 14,1 $\%$; en glándulas salivares mayores es del 15,3\%; el cáncer faríngeo abarca el $15 \%$ (orofaringe 5,7\%, laringofaringe $4,6 \%$, nasofaringe $4,5 \%$ ) y lugares sin especificar abarcan el otro $24,6 \%$. Es más frecuente en hombres que en mujeres y tiene su máxima incidencia en la quinta y sexta década de la vida.

Dentro de las causas del cáncer aparecen las influencias medioambientales, las cuales incluyen todos los factores no genéticos como la dieta, estilos de vida, ocupación, agentes infecciosos, contaminación ambiental, del aire, agua, suelo y alimentos (3).

Acerca de la alimentación, la OPS propone un plan de acción para la prevención de la obesidad en la niñez y la adolescencia en 2014. Una alimentación saludable debe basarse en el consumo diario de frutas y verduras, cereales integrales, legumbres, leche, proteínas con bajo consumo de carnes rojas y alimentos procesados. El efecto de los azúcares agregados en los alimentos procesados ha mostrado asociación estadística entre diabetes tipo 2 y síndrome metabólico y obesidad (15).

Según la OMS, la obesidad se duplicó el periodo de 1980 a 2008 a nivel mundial. En la Región de las Américas, la prevalencia del sobrepeso y la obesidad es más alta $-62 \%$ para el sobrepeso en hombres y mujeres y un $26 \%$ de obesidad en la población joven de 20 años de edad-. También se presenta un aumento de la prevalencia del sobrepeso y la obesidad en los niños de 0 a 5 años, de ambos sexos. Un informe reciente de siete países del Caribe Oriental indica que, entre el año 2000 y el año 2010, las tasas de sobrepeso y obesidad en los niños de 0 a 4 años, de ambos sexos, se duplicaron y pasaron de 7,4 \% en el 2000 a 14,8\% en el año 2010 (16).

De acuerdo con las anteriores cifras presentadas por la OMS, esta misma organización propone un seguimiento a las políticas de salud de la Región de las Américas para promover la educación y los conocimientos sobre la salud, en especial para las poblaciones con un bajo nivel de conocimientos sobre el cuidado de la salud, con el fin de aumentar la conciencia sobre la carga que imponen las enfermedades no transmisibles al sistema de salud pública nacional y la relación entre las enfermedades no transmisibles, la pobreza y el desarrollo social y económico de un país (16).

Según Orozco y Rojas (17), desde la psicología del deporte se pueden abordar los aspectos motivacionales que se producen al practicar algún deporte, para fortalecer la autoimagen o simplemente para aprovechar el tiempo libre. La actividad física y la recreación fomentan la comunicación, optimizan el rendimiento personal, la concentración y el liderazgo. En Colombia, desde el año 1995, se creó la Ley 181 por la cual se dictan disposiciones para el fomento del deporte, la recreación, el aprovechamiento del tiempo libre y la educación física y se crea el sistema nacional del deporte.

La actividad física se refiere a los movimientos corporales de cualquier tipo, que implican un gasto de energía mayor que el basal. La actividad física está dirigida a preservar la salud y mejorar las condiciones de vida de las personas, con el propósito de evitar el sedentarismo y la aparición de las enfermedades crónicas no transmisibles (17).

Respecto a las conductas sexuales de los adolescentes, se mencionan otros tantos factores de riesgo que conlleva la producción de enfermedades de transmisión sexual (ETS). Dentro de las ETS se menciona el síndrome de inmunodeficiencia adquirida, con especial atención en la adolescencia, que requiere una atención integral para lograr el equilibrio físico, psíquico y social en esta etapa del ciclo vital (18).

Dentro de las infecciones por virus, a nivel de los órganos reproductivos, está la infección por el Virus del Papiloma Humano (VPH), que es la infección de transmisión sexual con mayor prevalencia en la población sexualmente activa y es el factor etiológico de las lesiones pre neoplásicas y de cáncer invasivo de cuello uterino; entre más joven la población, la infección tiende a progresar a lesiones de alto grado y a cáncer (19).

La asociación entre VPH y cáncer cervico uterino se convierte en una de las mayores amenazas para la vida de los adolescentes, tomando como base que el periodo de latencia del virus sin producir ninguna sintomatología puede ser hasta de 10 años (20). Actualmente, el Virus del Papiloma Humano (VPH) se ha identificado como el agente causal necesario para el desarrollo del cáncer cervico uterino (20). 
De acuerdo con la encuesta de Demografía y Salud del año 2010 (21), el nivel de conocimiento de los adolescentes respecto al riesgo de adquirir una enfermedad de transmisión sexual (ETS) es muy bajo, especialmente entre las mujeres de 15 a 19 años de edad.

\section{Marco normativo}

La Ley 1388 de 2010 (22), tiene como objetivo "disminuir de manera significativa la tasa de mortalidad por cáncer en los niños y personas menores de 18 años, a través de la garantía por parte de los actores de la seguridad social en salud, de todos los servicios que requieren para su detección temprana y tratamiento integral, aplicación de protocolos y guías de atención estandarizados y con la infraestructura, dotación, recurso humano y tecnología requerida, en centros especializados habilitados para tal fin".

El Ministerio de la Protección Social, con la asesoría del Instituto Nacional de Cancerología (INC) y la Asociación Colombiana de Hematología y Oncología Pediátrica (ACHOP) diseñará, actualizará, y/o mejorará, según el anexo técnico de la Ley 1388, los requisitos esenciales de los centros de atención, los protocolos y las guías, para las causas más frecuentes de cáncer infantil en Colombia" (22).

La Ley 1388 de 2010 (22) establece los derechos de los niños, niñas y adolescentes con cáncer. "Cuando el médico tratante, independientemente de su especialidad, presuma la existencia de cáncer o de las patologías mencionadas, deberá remitir al paciente a la unidad de cáncer correspondiente a la zona, sin perjuicio de ordenar todos los exámenes de apoyo, diagnóstico y procedimientos especializados que se consideren indispensables hasta que el diagnóstico sea descartado".

Otro aparte de la Ley 1388 de 2010 (22) menciona que "a partir de la confirmación del diagnóstico de cáncer y hasta tanto el tratamiento concluya, los aseguradores autorizarán todos los servicios que requiera el menor, de manera inmediata".

\section{Plan de control del cáncer 2012- 2020}

La Organización Mundial de la Salud (OMS) propone un modelo de control del cáncer a nivel mundial que incluye el control del riesgo, la detección temprana, el tratamiento y rehabilitación y el cuidado paliativo como fines básicos para el control del cáncer a nivel mundial. Colombia es uno de los países que ha adoptado este modelo propuesto por la OMS (23).

En el mundo se presentan alrededor de 11 millones de casos nuevos de cáncer, de los cuales alrededor del 80 $\%$ se presenta en países en vías de desarrollo; significa que esta enfermedad, así como las enfermedades transmisibles, coexiste con necesidades básicas insatisfechas en una franja importante de la población (22).

El modelo conceptual para el control del cáncer se fundamenta en una visión amplia de la salud pública, que involucra acciones a nivel político, a nivel de servicios de salud y a nivel comunitario. El Plan Nacional para el Control del Cáncer en Colombia 2012- 2020 (23), busca desarrollar acciones para controlar los factores de riesgo, reducir la mortalidad evitable por cáncer y mejorar la calidad de vida de los pacientes con cáncer.

El Plan Nacional para el Control del Cáncer (23) se basa en los principios que expone el Plan Decenal de Salud Pública (24), como son la calidad, la eficiencia y la responsabilidad, que se orientan a la protección del derecho fundamental a la salud mediante los principios de universalidad, equidad y respeto a la diferencia, en el marco de la intersectorialidad y la participación comunitaria.

El Plan Nacional para el Control de Cáncer (23) define seis líneas estratégicas, de acuerdo a los objetivos del modelo conceptual: 1) Control del riesgo, prevención primaria, 2) Detección temprana de la enfermedad, 3) Atención, recuperación y superación de los daños causados por el cáncer, 4) Mejoramiento de la calidad de vida de pacientes y sobrevivientes de cáncer, 5) Gestión del conocimiento y la tecnología para el control del cáncer y 6) Formación del talento humano.

Se resalta aquí la estrategia de prevención primaria que está dirigida a proteger grupos vulnerables frente a los factores que pueden generar la enfermedad. La prevención primaria se dirige a responsabilizar al individuo y al grupo para colaborar en el desarrollo de su potencial para tomar decisiones con autonomía, en aras de mejorar las condiciones de vida (25).

La línea estratégica 1, prevención primaria del 
cáncer, se refiere al control del riesgo para reducir la incidencia de algunos tipos de cáncer y se han probado estrategias de promoción de la salud y protección específica, además de plantear diferentes metas: una de ellas, reducir la prevalencia de consumo de tabaco en jóvenes de 13 a 15 años por debajo del $18 \%$ (23).

Un estudio (26) realizado en las zonas urbanas marginadas de México, mediante una encuesta de seguimiento a 17.207 hogares, presenta los factores sociales y económicos que influyen en los jóvenes para tomar la decisión de fumar y consumir bebidas alcohólicas al mismo tiempo. Una de las mayores influencias la constituyó el ejemplo de ver jóvenes de mayor edad que bebían y fumaban en forma recíproca. Este estudió concluyó que los adolescentes que vivían con los padres tenían menor riesgo de iniciar el consumo de tabaco y alcohol a temprana edad.

Otra meta tiene que ver con la reducción de la prevalencia del tabaquismo en adultos de 18 a 69 años al $10 \%$ en los próximos 11 años. El hábito de fumar es un factor de riesgo para muchos tipos de cáncer. Tener uno o incluso varios factores de riesgo no significa que se desarrollará la enfermedad, porque se reitera que el cáncer es una enfermedad multifactorial. Dentro del control del riesgo, se tiene en cuenta la edad porque los factores de riesgo más comúnmente citados como tabaco, alcohol, componente hereditario, nutrición, virus, radiaciones y riesgos ocupacionales tienen un efecto acumulativo en el tiempo, lo que da consistencia al hallazgo epidemiológico de mayor prevalencia en adultos mayores (27)

- A continuación se enumeran las metas que plantea el Plan Nacional para el Control del Cáncer en Colombia 2012-2020 (23):

- Retrasar la edad de inicio de consumo del alcohol en los adolescentes colombianos por encima de 15 años.

- Lograr que el $100 \%$ de las empresas del sector formal que manejan los cinco elementos cancerígenos como son el asbesto, sílice, benceno, plomo compuesto inorgánico $\mathrm{y}$ radiación ionizante tengan niveles de exposición menores al valor límite permisible.

- Incrementar el consumo de frutas y verduras en la población general, en un $5 \%$.

- Incrementar la prevalencia de la actividad física global por encima del $42.6 \%$ en adultos entre 18 y 64 años, de acuerdo con el Plan Nacional de Salud Pública.

- Generar recomendaciones sobre la introducción de la vacuna contra el VPH dentro de la política del SGSSS 2012.

- Contar con la medición de la línea de base sobre los conocimientos y prácticas de la población colombiana respecto a la exposición a radiación solar ultravioleta para el año 2018.

El modelo para el control del cáncer incluye la movilización social como una herramienta fundamental para lograr el control de la enfermedad, no sólo del cáncer sino también de las enfermedades crónicas. El Instituto Nacional de Cancerología (INC) lidera un programa de revisión de experiencias internacionales y nacionales en movilización social para el control del cáncer (23).

Las diferentes estrategias mencionan intervenciones en diferentes sectores, que incluyen la participación comunitaria a través de redes de apoyo, por medio de fundaciones y alianzas, que propenden por el apoyo social y económico para los pacientes y familiares que atraviesan por esta dura enfermedad. Adicionalmente, el INC dispone de un inventario de organizaciones que actualiza periódicamente (23).

El Decreto 1757 de 1994 (28) establece las modalidades de participación social y comunitaria en salud. Se define la participación social como un "proceso de interacción social para intervenir en las decisiones de salud, respondiendo a intereses individuales y colectivos, basado en los principios de universalidad, solidaridad y equidad en la búsqueda de bienestar humano y desarrollo social". La participación comunitaria se ejerce en forma colectiva y corresponde al derecho que tienen las organizaciones comunitarias para participar en decisiones sobre los procesos de planeación, ejecución, evaluación y veeduría en salud.

La participación comunitaria en las instituciones del Sistema General de Seguridad Social en Salud (SGSSS) está reglamentada por medio de los
ISSN 1794-9831

E-ISSN 2322-7028

Vol. 13 No. 1

Ene - Jun 2016

Cúcuta, Colombia 
E-ISSN 2322-7028

Vol. 13 No. 1

Ene - Jun 2016

Cúcuta, Colombia
Comités de Participación Comunitaria (COPACO). El concepto de participación se refiere a espacios formalizados, con reglas definidas, donde se configuran compromisos articulados para la toma de decisiones y ejecución de proyectos.

Dentro de estas organizaciones, se mencionan algunas:

- Organizaciones populares: aquellas oficialmente establecidas que, en su mayoría, están integradas por afiliados y representan los intereses de grupos particulares de población. Entre éstas cabe citar a las organizaciones que representan a mujeres, niños o jóvenes, agricultores, desempleados, pueblos indígenas, ancianos y discapacitados.

- Organizaciones de diferentes profesionales: cuentan con afiliados que representan los intereses de personas que tienen la misma profesión o fuente de empleo. Las más importantes comprenden sindicatos y sus principales federaciones internacionales; asociaciones profesionales representativas de los trabajadores de la salud, la educación y el derecho, entre otros ámbitos profesionales; la comunidad científica y tecnológica; asociaciones o sindicatos de agricultores; cooperativas de productores.

- El sector privado: parte de la economía del país que no pertenece o no está controlada por el Estado. En el marco de la movilización social se debe sensibilizar al sector privado sobre su responsabilidad social frente al bienestar de la población y la de sus trabajadores. Existen industrias generadoras de factores de riesgo que tienen la obligación de minimizar sus impactos y de implementar soluciones en esta materia.

Por la relevancia de la participación ciudadana en el control del cáncer, se transcriben las acciones en el nivel comunitario que propone el Plan Nacional para el Control del Cáncer en Colombia 2012-2020 (23):

1. Desarrollo de estrategias de comunicación basadas en el modelo de comunicación para el control del cáncer desarrollado por el INC $\mathrm{y}$ orientadas a estimular el consumo de frutas y verduras y para desincentivar el consumo de tabaco y derivados.

2. Generación de alianzas con otros sectores para incentivar el consumo de frutas y verduras.

3. Desarrollo intersectorial con el Ministerio del Medio Ambiente para sensibilizar a la población sobre el índice de luz ultravioleta.

4. Impulso de estrategias para la actividad física en escenarios educativos, redes y grupos comunitarios y espacios laborales.

5. Información, comunicación, educación y vigilancia de los planes de prevención del cáncer en el marco de salud ocupacional

\section{Promoción de la salud, función esencial de la salud pública}

El cáncer es una enfermedad con grandes repercusiones sociales, económicas y emocionales a nivel individual y familiar. Dentro de las estrategias del Plan Nacional para el Control del Cáncer (23) está la prevención primaria, que comprende la protección específica con el fin de proteger a las poblaciones vulnerables de los factores que pueden deteriorar su salud. Dentro de estos factores está el riesgo de aparición del cáncer, que se describe como una problemática de salud pública que puede afectar a las personas sin importar su posición socioeconómica. De ahí la importancia de revisar el desarrollo de los estilos de vida saludable como una forma de prevención primaria del cáncer.

Retomando el concepto de promoción de la salud, dentro del marco conceptual del Plan Decenal de Salud Pública 2012-2021 (24), la promoción de la salud integra las acciones que debe liderar el territorio para modificar los determinantes sociales de la salud en cada región colombiana, para consolidar una cultura saludable que incluya: movilización social, formulación de políticas saludables, participación ciudadana y educación en salud.

La conceptualización de salud pública está íntimamente relacionada con la estrategia de Atención Primaria en Salud (APS) propuesta en la Conferencia Internacional de Alma ATA (29), con el lema Salud para todos en el año 2000. A lo largo del tiempo, la APS ha sido motivo de análisis y discusión, al punto 
que se ha utilizado esta estrategia para elaborar sistemas de salud con el fin de planear e implementar políticas públicas saludables.

La salud pública desarrolla funciones esenciales que equivalen al conjunto de actuaciones que se deben realizar, con fines concretos, para mejorar la salud de las poblaciones. Dentro de las once funciones esenciales de la salud pública se destaca la tercera, que corresponde a promoción de la salud. En el ámbito mundial se han convocado varias conferencias sobre promoción de la salud, entre ellas la Carta de Ottawa, en 1986 (30), que conceptualiza el término "Promoción de la salud: proceso mediante el cual las personas se empoderan y aumentan su control sobre los determinantes de la salud, para mejorar su salud".

La promoción de la salud, para su aplicación y difusión, se apoya en cinco áreas de acción interrelacionadas entre sí: construcción de políticas públicas saludables, creación de entornos o ambientes favorables, fortalecimiento de la acción comunitaria y la participación social, desarrollo de habilidades personales para el fomento de la salud y reorientación de los servicios de salud (30).

Las políticas públicas saludables tienen influencia directa en el desarrollo de programas de prevención, control de enfermedades y control de los determinantes de la salud. La creación de entornos saludables busca el mejoramiento de entornos o ambientes físicos, sociales, culturales y económicos, haciéndolos más amigables para aumentar la calidad de vida. La participación social busca crear espacios de concertación y negociación para fortalecer los derechos sociales de las personas y la comunidad. El desarrollo de las habilidades personales se relaciona con la educación para la salud y la reorientación de los servicios de salud busca reforzar actividades de educación sanitaria para influir en conductas de prevención y autocuidado (30).

El concepto de promoción de la salud es muy amplio y permite involucrar a todas las disciplinas de las ciencias de la salud. Desde la profesión de enfermería, la Ley 266 de 1996 (31), establece las funciones para el profesional de enfermería, dentro las cuales aparece la promoción de la salud en el individuo, la familia y la comunidad.

Hay una teórica de enfermería, Nola Pender, enfermera de la Universidad de Michigan reconocida por su Modelo de Promoción de la Salud, según el cual los determinantes de la salud y los estilos de vida se dividen en factores cognitivos y perceptuales; éstos últimos hacen referencia a las percepciones y conceptos que tienen las personas sobre la salud. Pender se apoya en el autocuidado y las relaciones armónicas con otros, incluido el medio ambiente, para crear ambientes favorables y la optimización de la vida (32)

\section{Acciones a nivel internacional}

En mayo del año 2008 se realizó en Ginebra, Suiza, la $61^{\text {a }}$ Asamblea Mundial de la Salud (33), donde se examinaron aspectos de salud pública dentro de los cuales figuran las enfermedades cardiovasculares y el cáncer, por ser éstas las principales causas de muerte en el mundo.

La OMS, en conjunto con la Organización de las Naciones Unidas para la Alimentación y la Agricultura (FAO) (34), en mayo de 2002, realizó la Consulta de Expertos de la OMS y de la FAO, con el tema puntual de la relación entre la nutrición y la prevención de enfermedades crónicas no transmisibles. De esta reunión de expertos surgió un informe, en el año 2003, el cual señala que una dieta saludable basada en la ingesta abundante de frutas y verduras, así como el ejercicio físico, son los pilares para combatir las enfermedades crónicas. Este informe fue el soporte para la implementación de las políticas de alimentación y ejercicio físico en Colombia y los países de la Región Andina.

La OMS adelanta, a nivel mundial, un plan para fortalecer la movilización social respecto a la mejora y el incremento de la seguridad alimentaria y nutricional que garantice la producción y la comercialización de las frutas en diferentes regiones del mundo. Para el caso colombiano, en el año 2012 se realizó el VIII Congreso Mundial de Promoción al Consumo de Frutas y Verduras (35), para controlar las enfermedades no transmisibles, en cooperación con el Foro Económico Mundial.

Hay muchos factores que influyen directamente en el desarrollo nutricional de los niños; los más sobresalientes son la pobreza, la falta de agua potable y las condiciones favorables de saneamiento básico. La OMS establece el plan de acción a nivel regional, con énfasis en la nutrición, la salud y el desarrollo

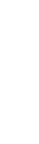


y se resaltan, en este documento, las inequidades existentes en la región de las Américas: se calcula que la pobreza en esta parte del mundo afecta a 213 millones de personas (36).

El crecimiento y desarrollo sano de los niños es la base del desarrollo humano. La desnutrición crónica es más frecuente entre las poblaciones indígenas, puesto que en estos grupos no hay oferta de servicios de salud por dificultades en el transporte debido a la dispersión geográfica, escases de vías de comunicación y barreras culturales entre las creencias y las prácticas en el cuidado de la salud, afectando principalmente a los niños y a las madres gestantes(37).

Continuando con la revisión del control del cáncer en América Latina, se encontraron dificultades en la detección temprana de la enfermedad por no buscar ayuda médica cuando aparecen los síntomas y por diferentes razones como la dificultad de remisión a los servicios médicos especializados. Esto contribuye a que la enfermedad se descubra en fase avanzada, especialmente el cáncer de mama, de cuello uterino y gástrico. Para el cáncer de pulmón, el estudio diagnóstico requiere sistemas de imagen de alta calidad y la mayoría de las áreas no posee capacidad para realizar estos exámenes, lo que constituye un obstáculo para la detección temprana.

La OMS propuso una estrategia y plan de acción para la prevención y control del cáncer cérvico uterino en América (38), basada en la evaluación inicial de los programas existentes y la evaluación de la carga de infecciones por el VPH en población joven y adolescente. Esta iniciativa buscó fortalecer las acciones de información y educación, en especial a las mujeres indígenas, de grupos desfavorecidos y vulnerables, de zonas rurales y trabajadoras sexuales.

Revisando antecedentes al respecto en los países de la Región Andina, en Chile, por ejemplo, se establece la estrategia de Promoción de Salud desde 1997 y se creó el Consejo Nacional de Promoción de Salud en 1998 (39), cubriendo 341 comunas del país, en cada de las cuales se desarrollaron proyectos de dieta saludable y ejercicio físico.

Por su parte, la Coalición Multisectorial contra el Cáncer, en Perú, con participación del sector público y privado, elaboró el Plan Estratégico Perú 20062016 (40) contra el cáncer, sobre el cual "se hace un diagnóstico situacional; se identifican las necesidades de acceso, se define el compromiso de las instituciones; se define la visión y misión, los ocho lineamientos estratégicos y se concluye que se debe elaborar e implementar un Plan Nacional de Prevención y Control del Cáncer dentro del marco normativo y jurídico del Ministerio de Salud y del Estado". Aquí se fortaleció la promoción de la salud, orientada a la "prevención del cáncer mediante información, educación y comunicación, se mejoró y acercó la oferta de servicios mediante la desconcentración y descentralización de la atención oncológica" (40).

En cuanto a Cuba, hay que centrarse en las paradojas y controversias en relación con el consumo de tabaco, que tienen como resultado la brecha existente en el discurso y la realidad. Existe en este país un Programa de Prevención y Control del Tabaquismo concebido con carácter nacional, pero el arraigo al hábito y la cultura de un pueblo fumador es muy fuerte en el pueblo cubano. "La salud pública tiene un importante reto, pues la situación que se muestra hoy día en relación con otros países impone que se asuma con total responsabilidad la política y estrategia documentada" (41).

Al respecto de la actividad física en el adulto mayor en Cuba, Rodríguez (42), refiere que hay satisfacción de la mayoría de los adultos mayores por las actividades en que pudieron participar. También afirma que la "actividad física es la clave de la salud, con un balance nutricional en sus principios inmediatos y unos ejercicios bien dosificados y practicados, los años no se detendrán pero el proceso de envejecimiento será más lento" (42).

\section{Estrategias a nivel comunitario en Colombia}

El Plan Nacional para el Control del Cáncer en Colombia (2012-2020) (23) tiene como propósito posicionar, en la agenda pública, el cáncer como un problema de salud pública y movilizar la acción del Estado, la acción intersectorial, la responsabilidad social empresarial y la corresponsabilidad individual para el control del cáncer en el país. Para cumplir este propósito, el plan define seis líneas estratégicas y en cada una se enuncian acciones a nivel político, a nivel comunitario y a nivel de los servicios de salud (23).

Un tema que es de interés general se refiere al cuidado al medio ambiente. Los profesionales de la salud tienen una concepción del cuidado integral, incluyendo los factores ambientales. La salud 
ambiental no se limita solamente a los ambientes físicos, químicos o biológicos sino que tiene que ver con ambientes domésticos, escolares, sociales, culturales y económicos que son susceptibles de interactuar los unos con los otros (43).

Se describen algunas estrategias en promoción de la salud para fortalecer las capacidades de las personas respecto al cambio de comportamientos en los estilos de vida. Giraldo et al.(44) ratifican la promoción de la salud, que se consigue a través de los mecanismos intrínsecos o internos de las personas, que llevan al autocuidado y a las acciones de ayuda mutua que realizan las personas según las situaciones que estén vivenciando. Esto propicia la educación en salud a través de la participación comunitaria.

Es importante resaltar que, dentro de las estrategias de prevención primaria para las enfermedades crónicas no transmisibles, es fundamental el enfoque integrado de prevención, detección temprana y tratamiento, según el actual director de la Agencia Internacional para la Investigación en Cáncer, porque se corrobora que el $50 \%$ de los cánceres se pueden prevenir, de acuerdo con el informe mundial de cáncer 2014 (45).

Para que un plan de acción en promoción de la salud sea efectivo, se requiere de un personal de salud comprometido. Coral y Vargas (46) manifiestan que en la formación de futuros profesionales de enfermería se deben incluir los estilos de vida saludables para aplicarlos en el diario vivir. Lo ideal es revisar la aplicación de los estilos de vida y evaluarlos, porque la mayoría de los estudiantes universitarios conoce los beneficios de los estilos de vida, pero en la realidad, por diferentes factores, no los pone en práctica.

Frente a la carga tan alta que genera el cáncer, en el aspecto físico, emocional, familiar, social, económico y laboral, surge una respuesta social desde diversas organizaciones que apoyan a los pacientes con cáncer. Estas entidades desempeñan una labor importante en la defensa y en la difusión de los derechos fundamentales de los pacientes con cáncer. El INC dispone de un control e inventario de estas organizaciones y periódicamente lo actualiza. También existen organizaciones y fundaciones que apoyan no sólo a los pacientes sino a sus familias, como es el caso de las fundaciones que apoyan a los niños con cáncer (23).

Estas organizaciones se caracterizan por ser sin ánimo de lucro y algunas desarrollan iniciativas con el fin de recolectar fondos y apoyar a los pacientes. Una de estas iniciativas se refiere a los programas de reciclaje: estas organizaciones venden a empresas recicladoras el material, con el fin de conseguir recursos económicos para apoyar en forma integral a niños, niñas y adolescentes que padecen cáncer.

Desde la promoción de la salud se procura el cuidado integral de las personas en su entorno; en este caso, el cuidado al medio ambiente es de interés para todos y para ello surgen iniciativas de participación comunitaria con el propósito de apoyar programas de ayuda a personas con cáncer. Se revisaron experiencias en diferentes sectores sobre el tema de reciclaje.

En este sentido, en Caracas, Ponte (47) reporta los beneficios de un programa de reciclaje a nivel escolar, que inicia con el papel y poco a poco va instaurando la cultura del reciclaje para incluir otros productos reciclables como el plástico, el cartón y el vidrio, máxime si se tiene en cuenta que el relleno sanitario de Caracas ya está al límite de su capacidad.

En México se analizan los riesgos para la salud y al ambiente que se producen por no implementar programas de reciclaje. Cristan et al. (48), refieren la problemática que genera el material plástico en los botaderos y rellenos sanitarios, porque este material no se degrada y lo ideal es volver a reutilizarlo.

En Colombia también se adelantan diversas alternativas para contribuir al cuidado del medio ambiente. Quintero et al. (49), en Sogamoso, exponen la implementación de un programa de reciclaje a nivel escolar, denominado Econcierto, que es una herramienta pedagógica que instruye sobre los temas puntuales de selección y reciclaje de material plástico.

Los programas de reciclaje son llamativos desde la integralidad, porque si los estudiantes comprenden los procesos que se deben desarrollar para la reutilización de los materiales, bien sea plástico, vidrio o cartón, con mayor facilidad van a colaborar y difundir dichos mecanismos de reutilización. (50)

El programa de reciclaje, ha tenido gran acogida en comunidades educativas, jardines, colegios y universidades. Se revisa el aporte donación de la Fundación Universitaria del Área Andina (FUAA) a

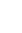


una de estas organizaciones, en el periodo 2011 a 2015, en el siguiente cuadro resumen:

Ene - Jun 2016

\begin{tabular}{|c|c|}
\hline AÑo & $\begin{array}{c}\text { MATERIAL PLÁSTICO } \\
\text { (Tapas) }\end{array}$ \\
\hline 2012 & 1.500 Kilos \\
\hline 2013 & 3.450 Kilos \\
\hline 2014 & 2.001 .5 Kilos \\
\hline 2015 & 1.550 Kilos \\
\hline TOTAL & $\mathbf{8 . 5 0 1 . 5}$ Kilos \\
\hline
\end{tabular}

Fuente: Certificados de donación al programa de reciclaje, para apoyar a los niños con cáncer.

\section{CONCLUSIONES}

La promoción de la salud constituye una estrategia de difusión y de educación para la comunidad en general. Las organizaciones que apoyan a los pacientes con cáncer, realizan una labor significativa porque logran una movilización importante frente a esta problemática de salud pública.

Las estrategias propuestas en conferencias internacionales desde APS y promoción de la salud, en Ottawa, Canadá, cobran vigencia para fortalecer conductas de autocuidado y estilos de vida saludable para adelantar programas de prevención primaria de cáncer a nivel de instituciones de educación superior. Cuando estas acciones se complementan con programas de las organizaciones especializadas para proporcionar ayuda a pacientes con cáncer, se fortalece la movilización social. Esto es lo que se pretende al relacionar el material entregado en donación, porque es una evidencia que soporta las intervenciones de prevención primaria del cáncer en forma integral, con la participación comunitaria.

Las enfermedades crónicas no transmisibles tocan todos los estratos y todos los grupos sociales, culturales y religiosos. Desde las diferentes ópticas con que se mire, lo ideal es estar informados, conocer signos y síntomas, los factores de riesgo y también los factores protectores para implementar cultura de vida saludable, ahondar en los conocimientos y difundirlos a otros para instaurar espacios de reflexión y análisis en el marco de las estrategias de prevención primaria.

\section{CONFLICTO DE INTERESES}

La autora declara no tener ningún conflicto de intereses. 


\section{REFERENCIAS BIBLIOGRÁFICAS}

1. Organización Mundial de la Salud (OMS). Plan de acción mundial para la prevención y el control de las enfermedades no transmisibles 2013-2020. [Internet]. OMS; 2013 [consultado 10 de abril de 2015]. Disponible en: http://www.who.int/cardiovascular_diseases/15032013_updated_revised_draft_action_plan_spanish.pdf

2. Organización Panamericana de la Salud (OPS), Organización Mundial de la Salud (OMS). Plan de acción para la prevención y el control de las enfermedades no transmisibles en las Américas 2013-2019. Washington, DC: OPS; 2014

3. Sierra-Lopez A, Saenz-González M, Fernández-Crehuet J, Salleras-Sanmmartí L,Cueto-Espinar A, GestalOtero J et al. Medicina preventiva y salud pública. Barcelona España Editorial Elsevier Masson; 2008. p 838.

4. Molina MG, Ramirez GA, Ruiz, GA. Tensiones en las decisiones en Salud Pública en el sistema de salud colombiano: El bien común en confrontación con los intereses y prácticas particulares. Medellín: Universidad de Antioquía., Pulso \&Letra Editores; 2014. p. 41.

5. Organización Panamericana de la Salud (OPS). Salud en las Américas. Edición 2012. [Internet]. Washington (D.C.): OPS; 2012 [consultado 13 de febrero del 2014]. Disponible en: http://www.paho.org/saludenlasamericas/ index.php?option $=$ com_content $\&$ view $=$ article\&id=9\&Itemid=14\&lang=es

6. Pardo-Ramos C, Cendales-Duarte R. Incidencia, mortalidad y prevalencia de cáncer en Colombia 2007-2011. $1^{\mathrm{a}}$ ed. v.1. [Internet]. Bogotá. D.C.: Instituto Nacional de Cancerología; 2015 [consultado 24 de marzo de 2016 ]; p. 148. Disponible en: http://www.cancer.gov.co/files/libros/archivos/incidencia1.pdf

7. Alcaldía Mayor de Bogotá. Secretaría Distrital para la mujer. Observatorio de mujer y género. Mortalidad de mujeres por afecciones cancerígenas en Bogotá. Día mundial de la lucha contra el cáncer. [Internet]. 2016 [consultado 8 de marzo de 2016]. Disponible en http://www.sdmujer.gov.co/inicio/899-mortalidad-de-mujerespor-afecciones-cancerigenas-en-bogota-dia-mundial-de-la-lucha-contra-el-cancer

8. American Cancer Society. ¿Cuáles son los factores del cáncer de seno?. [Internet]. 2014 [consultado 3 de febrero de 2016]. Disponible en: http://www.cancer.org/espanol/cancer/cancerdeseno/resumen/resumen-sobre-elcancer-de-seno-causes-what-causes

9. Romero-Figueroa MS, Santillán-Arreygue L, Olvera-Hernández PC, Morales-Sánchez MA, Ramírez-Mendiola VL. Frecuencia de factores de riesgo de cáncer de mama. Ginecol Obstet Mex [Internet]. 2008 [consultado 10 de febrero de 2016]; 76(11): 667-72. Disponible en: http://www.medigraphic.com/pdfs/ginobsmex/gom-2008/ gom0811f.pdf

10. Aguilar-Cordero MJ, Neri-Sánchez M, Padilla-López CA, Pimentel-Ramírez ML, García Rillo A, SánchezLópez AM. Factores de riesgo como pronóstico de padecer cáncer de mama en un estado de México. Nutr. Hosp. [Internet]. 2012 [consultado 2 de febrero de 2016]; 27(5): 1631-1636. Disponible en: http://scielo.isciii.es/ scielo.php?pid=S0212-16112012000500038\&script=sci_arttext

11. República de Colombia. Ministerio de la Protección Social. Ley 1355 de 2009, octubre 14, por medio de la cual se define la obesidad y el control de las enfermedades crónicas no transmisibles asociadas a esta como una prioridad de salud pública y se adoptan medidas para su control, atención y prevención. Diario Oficial No 47.502 del 14 de octubre de 2009. [Internet]. Bogotá D.C.: Gobierno Nacional: 2009 [consultado 2 de febrero de 2016]. Disponible en: http://www.icbf.gov.co/cargues/avance/docs/ley_1355_2009.htm

12. Pardo C, Cendales R. Incidencia estimada y mortalidad por cáncer en Colombia, 2002-2006. v.1. 1ª ed. Bogotá. D.C.: Instituto Nacional de Cancerología; 2010. p. 143

13. Serena-Gómez E, Bologna-Molina RE, Nevarez-Rascón A, Rocha-Buelvas A. Prevalencia del VPH en el proceso de malignización de lesiones de vías aérodigestivas superiores. Int. J. Odontostomat. [Internet]. 2011 [consultado 2 de febrero de 2016]; 5(1): 5-12. Disponible en: http://www.scielo.cl/scielo.php?pid=S0718381X2011000100001\&script=sci_arttext

14. Organización Panamericana de la Salud (OPS), Organización Mundial de la Salud (OMS). Unidad 2: Salud y enfermedad en la población. En: OPS, OMS. Módulo de principios de epidemiología para el control de enfermedades (MOPECE). $2^{\mathrm{a}}$ ed. Revisada. [Internet]. [consultado 2 de noviembre de 2015]. Washington, D.C.: OPS; 2011 Disponible en: http://myslide.es/documents/mopeceespmod02atualpdf.html

15. Organización Panamericana de la Salud (OPS). Organización Mundial de la Salud (OMS). Plan de acción para la prevención de la obesidad en la niñez y la adolescencia. [Internet]. Washington D.C.: OPS/OMS; 2014 [consultado 8 de febrero de 2015]. Disponible en : http://www.paho.org/hq/index.php?option=com_docman\&task=doc_vie w\&Itemid=270\&gid=28899\&lang=es]

16. Organización Mundial de la Salud (OMS). Seguimiento de la reunión de alto nivel de la Asamblea General de las Naciones Unidas para llevar a cabo un examen y una evaluación generales de los progresos realizados en la 
prevención de las enfermedades crónicas no transmisibles. [Internet]. 2014 [consultado 15 de Octubre de 2015] Disponible en: http://apps.who.int/gb/ebwha/pdf_files/EB136/B136_11-sp.pdf

17. Orozco AJ, Rojas JE. Motivación hacia programas de ejercicio, deporte y actividad física de los empleados de UNE telefónica de Pereira. 2013. [Tesis en internet]. Bogotá: Universidad Tecnológica de Pereira; 2013 [consultado 2 de febrero de 2016] Disponible en: http://repositorio.utp.edu.co/dspace/bitstream/11059/3425/1 179606986132O74.pdf

18. Pérez-Morffi L, Santos-Blanco MA, Paz-Perez Y. Comportamiento de las infecciones de transmisión sexual en adolescentes. Acta Médica del Centro 2014; 8 (3).

19. Barbón-Pérez OG. Fuentes de información sobre educación sexual en adolescentes estudiantes de enfermería. Revista Cubana de Higiene y Epidemiología 2011; 49(2): 238-246.

20. Muñoz N, Bravo LE. Epidemiology of cervical cáncer in Colombia. Salud pública Méx [Internet]. 2014 [consultado 12 agosto de 2014]; 56(5): 431-439. Disponible en: http://www.scielosp.org/scielo.php?script=sci_ arttext\&pid=S0036-36342014000500010\&lng=en\&tlng

21. Asociación Probienestar de la Familia Colombiana (Profamilia). Encuesta nacional de demografía y salud ENDS 2010 Colombia. [Internet]. Bogotá D.C.: Profamilia; 2010 [consultado 19 de Septiembre de 2014]. Disponible en: http://pdf.usaid.gov/pdf docs/Pnady407.pdf

22. Congreso de la República. Ley 1388 de 2010, mayo 26, por el derecho a la vida de los niños con cáncer en Colombia. Diario Oficial 47.721 de mayo 26 de 2010. Bogotá D.C.: Gobierno Nacional; 2010.

23. República de Colombia. Ministerio de Salud y Protección Social. Instituto Nacional de Cancerología. Plan Nacional para el Control del Cáncer en Colombia (2012-2020) [Internet]. Bogotá D.C.: Minprotección; 2012 [consultado 3 de agosto de 2014]. Disponible en www.minsalud.gov.co/Documentos\%20y\%20Publicaciones/ Plan\%20nacional\%20para\%20el\%20control\%20del\%20cáncer\%20en\%20Colombia.pdf

24. República de Colombia. Ministerio de la Protección Social. Plan Decenal de Salud Pública 2012-2021. Bogotá D.C.: Ministerio de Salud y Protección Social; 2012.

25. Martínez-Hernández J. Nociones de salud pública. Madrid, España: Editorial Díaz de Santos; 2013.

26. Urquieta JE, Hernández-Ávila M, Hernández B. El consumo de tabaco y alcohol en jóvenes de zonas urbanas marginadas de México. Un análisis de decisiones relacionadas. salud pública de méxico 2006; 48 (Supl 1):s30-s40.

27. Rocha-Buelvas A. Cáncer oral: el papel del odontólogo en la detección temprana y control. Rev Fac Odontol Univ Antioq 2009 21(1):112-121

28. República de Colombia. Ministerio de Salud. Decreto 1757 de 1.994, agosto 3, por el cual se organizan y establecen las modalidades y formas de participación social en la prestación de servicios de salud, conforme a lo dispuesto en el numeral 11 del artículo 4o del Decreto Ley 1298 de 1994. Diario Oficial 41477 de agosto 05 de 1994: Bogotá D.C.: Ministerio de Salud; 1994.

29. Organización Mundial de la Salud (OMS). Declaración de Alma-Ata. Conferencia Internacional sobre Atención Primaria de Salud; Alma-Ata, USSR, del 6 al 12 de septiembre de 1978. [Internet]. Ginebra: OMS; 1978 [consultado 13 de septiembre de 2014]. Disponible en: http://www.paho.org/spanish/dd/pin/almaata declaration. htm. 7.

30. Organización Mundial de la Salud (OMS). Carta de Ottawa para la Promoción de la Salud. Primera Conferencia Internacional sobre Promoción de la Salud; 21 de CD53/10, Rev. 113 noviembre de 1986. [Internet]. Ottawa, Ontario (Canadá): OMS; 1986 [consultado 13 de marzo de 2014]. Disponible en: http://www1.paho.org/spanish/ HPP/OttawaCharterSp.pdf

31. República de Colombia. Ministerio de Salud. Ley 266 de 1.996, enero 25, por la cual se reglamenta la profesión de enfermería en Colombia y se dictan otras disposiciones. Diario Oficial No 42.710 Bogotá D.C.: Ministerio de Salud; 1996.

32. Giraldo-Osorio A, Toro Rosero MY, Macias-Ladinoa M, Valenci-Garcés CA, Palacio-Rodríguez S. La promoción de la salud como estrategia para el fomento de estilos de vida saludable. Revista Hacia la Promoción de la Salud 2010 15(1):128-143.

33. Organización Mundial de la Salud (OMS). 61a Asamblea Mundial de la Salud. Centro de Prensa. 19-24 mayo de 2008. [Internet]. 2008 [consultado 9 de diciembre de 2008]. Disponible en: http://www.who.int/mediacentre/ events/2008/wha61/es/

34. Organización Mundial de la Salud (OMS). Serie de Informes Técnicos 916. Dieta, nutrición y prevención de enfermedades crónicas. Informe de una Consulta Mixta de Expertos OMS/FAO. [Internet]. Ginebra: OMS; 2003 [consultado 2 de septiembre de 2013] Disponible en: ftp://ftp.fao.org/unfao/bodies/coag/coag18/j1251s.pdf

35. República de Colombia, Ministerio de la Protección Social. Organización de las Naciones Unidas para la Alimentación y la Agricultura (FAO). VIII Congreso Mundial de Promoción al Consumo de Frutas y verduras 
para el control de las enfermedades no transmisibles y la seguridad alimentaria. Colombia. [Internet]. Bogotá; Minprotecciòn-Fao; 2012 [consultado 21 de noviembre de 2015]. Disponible en: http://www.osancolombia.gov. co/doc/MemoriasVIIICongreso\%20Mundial\%20_\%20Promoci\%C3\%B3n_frutas_hortalizas_Colombia_2012. pdf

36. Organización Mundial de la Salud (OMS), Organización Panamericana de la Salud (OPS). Estrategia y plan de acción regionales sobre la nutrición en la salud y el desarrollo, 2006-2015. $47^{\circ}$ Consejo Directivo $58^{\mathrm{a}}$ Sesión del Comité Regional. [Internet]. Washington, D.C., OMS, OPS; 2006 [consultado 12 de marzo de 2014] Disponible en: http:/www.paho.org/hq/dmdocuments/2009/estrategia-regional-nutricion-esp

37. Fondo de las Naciones Unidas para la Infancia (UNICEF). Estrategia de UNICEF en América Latina y el Caribe para contribuir a la reducción de la morbimortalidad materna, neonatal e infantil. 2011-2015. Salud y Nutrición Informe. [Internet]. Panamá: UNICEF; 2010 [consultado 30 septiembre de 2014]. Disponible en http://www. unicef.org/lac/Estrategia_Mortalidad_Materna_Neonatal.pdf

38. Organización Panamericana de la Salud. Estrategia y plan de acción regional para la prevención y el control del cáncer cervico uterino en América Latina y el Caribe. [Internet]. Washington: OPS; 2010 [consultado 3 de octubre de 2014]. Disponible en: http://www.rho.org/files/PAHO_Regional_Strategy_2010_sp.pdf

39. Crovetto M, Vio F. Antecedentes internacionales y nacionales de la promoción de la salud en Chile. Lecciones aprendidas y proyecciones futuras. Rev. chil. nutr. [Internet]. 2009 [consultado 15 de junio de 2014]; 36 (1):3245. Disponible en: http://www.scielo.cl/scielo.php?pid=S0717-75182009000100004\&script=sci_arttext

40. Pinillos-Ashton L, Limache-García A. Contribuciones de la Coalición Multisectorial Perú contra el Cáncer. Rev. Perú. med. exp. salud publica ene. 2013; 30 (1):99-104.

41. Suarez-Lugo N. Paradojas, controversias, discurso y realidad del tabaquismo. Revista Cubana de Salud Pública 2011;37(1): 120-131.

42. Rodríguez-Rojas A. Actividades físicas recreativas para favorecer el estilo de vida saludable del adulto mayor perteneciente al CDR 1 de la zona 66 del consejo popular Roberto Rivas Fraga. EFDeportes .com Revista digital [Internet]. 2010 [consultado 27 de agosto de 2015]; 15(149). Disponible en: http://www.efdeportes.com/efd149/ estilo-de-vida-saludable-del-adulto-mayor.htm

43. Instituto Nacional de Salud Pública. Salud Pública. Teoría y práctica. México D.F.: Editorial El Manual Moderno, S.A. de C.V.; 2013.

44. Giraldo A, Toro MY., Macias AM, Valencia CA, Palacio S. La promoción de la salud como estrategia para el fomento de estilos de vida saludables. Hacia la Promoción de la Salud. [Internet] 2010 [consultado 10 de febrero de 2015]; 15(1):128-143. Disponible en: http://www.scielo.org.co/pdf/hpsal/v15n1/v15n1a09.pdf

45. Agencia internacional para la investigación del cáncer. (IARC) Conferencia: Global Health Lecture, coorganizada por IS Global, Cresib y Creal Wild, C. 21 de febrero de 2014. [Internet]. [consultado 2 de abril de 2016] Disponible en: http://www.isglobal.org/-/la-prevencion-evitaria-el-50-de-los-canceres-en-el-mundo.

46. Coral RC, Vargas LD. Estrategias que promueven estilos de vida saludables. rev.udcaactual.divulg.cient. [Internet]. 2014 [consultado 1 de febrero de 2016]; 17(1): 35-43. Disponible en: http://www.scielo.org.co/scielo. php?script $=$ sci_arttext\&pid=S0123-42262014000100005

47. Ponte-Chacín $\bar{C}$. Manejo integrado de residuos sólidos: Programa de reciclaje Instituto Pedagógico de Caracas. Revista de Investigación [Internet]. 2008 [consultado 3 de marzo de 2014]; 32(63): 173-200. Disponible en: http://www.scielo.org.ve/scielo.php?script=sci_arttext\&pid=S1010-29142008000100010

48. Cristan A, Ize I, Gavilán A. La situación de los envases de plástico en México. Gaceta Ecológica [Internet]. 2001 [consultado 13 de junio de 2015]; (69): 67-82. Disponible en http://www.redalyc.org/pdf/539/53906905.pdf

49. Quintero-Bonilla DL. Plan de negocios para una empresa de reciclaje plástico. [Internet]. Bogotá D.C., Colombia: Universidad Javeriana; 2014 [consultado 6 de septiembre de 2014]. Disponible en: http://repository.javeriana. edu.co/handle/10554/15778

50. Castañeda-Heredia LF, Universidad EAFIT. Artículos de los proyectos de grado realizados por los estudiantes de ingeniería mecánica que se graduaron en el año 2004. Cuadernos de Investigación. [Internet]. 2005 [consultado 25 de septiembre de 2014]; 32. Disponible en: http://publicaciones.eafit.edu.co/index.php/cuadernos-investigacion/ article/view/1312/1183 